\title{
IN THE CONTEXT OF TIME-INDEPENDENT PARAMETERS IN TWO QUANTUM SYSTEMS: QUANTUM ENTANGLEMENT AND CORRELATIONS WITH NEGATIVITY MEASUREMENT
}

\author{
Rasim DERMEZ ${ }^{1,2}$ *(D) , Yılmaz TUNÇER ${ }^{3}$ \\ ${ }^{1}$ University of Afyon Kocatepe, Arts and Science Faculty, Department of Physics, Afyonkarahisar, Turkey \\ ${ }^{2}$ University of Afyon Kocatepe, Afyon Vocational School, Afyonkarahisar, Turkey, \\ ${ }^{3}$ University of Uşak, Arts and Science Faculty, Department of Mathematics, Uşak, Turkey
}

\begin{abstract}
In this paper, we consider two phonons and three-level trapped ion in $\Lambda$ configuration forming Hilbert 12-space. The negativity and quantum correlations are revealed in trapped ion two phonon states system. Three values of LDP, $\eta=0.006$,

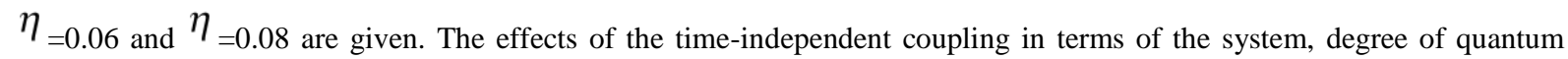
entanglement are investigated. Therefore, we have found the main optimal times for obtaining the high amount of entanglement with negativity.
\end{abstract}

Keywords: Interaction between ion and phonons, Reduced density matrix, Probability amplitudes, Entanglement

\section{INTRODUCTION}

In quantum mechanics, quantum states as usual are evident in itself with laws [1]. Entangled states are the proper kind of quantum correlation between two quantum system. Entanglement is an attractive physical phenomenon in which the overlap of two separable states is can be entangled state with photons. The widely read Einstein, Podolsky and Rosen (EPR) paper, contrary to what is known, has actually been published to criticize quantum mechanical laws [2]. In the same year, N. Bohr published a paper [3] with alike this EPR paper. The prominent article presented the entanglement with conversations on quantum theory. For the quantum theory, 1935 was an interesting year. In Erwin Schrödinger's article in Naturwissenschaften introducing "Verschränkung", where he advocated quantum theory [4]. Quantum measurement is discussed a local physical process [5]. Trapped ions systems are important for the entangled states works [6, 7, 8], concurrence $\mathrm{C}$ [9]. It has reported an applying entanglement created the Exchange interaction for many quantum information processing [10].

Quantum dynamics of ionic-phononic system with respect to quantum entropy $\mathrm{E}$ is investigated by R.

Dermez et al. [11]. The deep Lamb-Dicke regime (LDR) described with LDP of small, $\eta<<1$, such as this study. LD limit is not accordingly established with common experiments [12]. Such a way experiments act in named as beyond LDR here $\eta<1$, for examples $\eta \sim 0.2$ [13]. Entanglement of qutrit states [14] are testified by a quantum system for lower order terms of density matrix.

Product base and entangled base are shown generalization of Schmidt coefficients [15]. Quantum entanglement of states of pure qutrit [16] and of states of mixed qubit-qutrit [17] are demonstrated by a quantum system within the $\Lambda$ configuration. Advanced calculations and results in the four articles

*Corresponding Author: dermez@aku.edu.tr

Received: 20.03.2020 Published: 31.08.2020 
mentioned in [14-17] contain differences from classical theoretical calculations. Measurement of entropy is achieved for states of qutrit [18].

Quantum entanglement measurements are used to determine any known state is separable or entangled. Therefore, $\mathrm{C}$ and $N$ are offered for pure states [19]. Quantum computation processes in trapped ions are common in phonon nonclassical cases. For Fock states, squeezed [20], coherent states of odd-even [21], and their superpositions [22] were suggested. $N$ is a entanglement measure that a useful characterization in quantum information, commonly in ionic system. We report analytical results of quantum entanglement for system via $N$ for the LDR and12-D of Hilbert space. We focus the quantum correllations in $N[11,16]$ with respect to the total and the reduced density matrix. With respect to Ref. [16] we illustrated these evolutions of $N$ in the Figures. 2-4 for trapped ion two phonons system.

The rest of the study is coordinated as follows. Section 2 discusses growth for two unentangled qubits and analitical solutions in the quantum system. Section 3 describes how to obtain highly negativity of system by the LDR. The results and comments are given in Section 4.

\section{A SOLUTION OF TRAPPED ION-PHONONS SYSTEM AND ITS MODEL}

We propose a trapped ion with two phonons in Hilbert 12-space. The quantum dynamics of trapped ionphonons system is emerged by previous investigation [23,24]. The Hamiltonian of system is $H_{\text {total }}=H_{i o n}+H_{1}+H_{2}$, and $H_{i o n}$ indicates Hamiltonian of trapped ion-phonons system:

$$
H_{\text {ion }}=\omega_{g}|g\rangle\left\langle g\left|+\omega_{r}\right| r\right\rangle\langle r|+0| e\rangle\langle e|+\frac{p_{x}^{2}}{2 m}+\frac{1}{2} m v^{2} x_{\text {ion }}^{2} .
$$

The e-level energy is $\omega_{e}=0$, r-level is $\omega_{r}$, and g-level is $\omega_{g}$. Here $H_{1}$ and $H_{2}$ are Hamiltonians of these interactions for excited-ground and excited-raman:

$$
\begin{gathered}
H_{e-g}=H_{1}=\frac{\Omega}{2} e^{i\left(k_{1} x_{i o n}-\omega t\right)}|e\rangle\langle g|+\text { hermitian } c . \\
H_{e-r}=H_{2}=\frac{\Omega}{2} e^{i\left(-k_{2} x_{i o n}-\omega t\right)}|e\rangle\langle r|+\text { hermitian } c .
\end{gathered}
$$

where $\square=1, p_{x}$ and $x_{i o n}$ are momentum and the $x$-component of position of ion center of mass movement. The movement of ion in the system is along the $x$-axis (one-D). Atomic levels are shown: $|e\rangle \rightarrow_{\text {trapped ion excited level, }}|r\rangle \rightarrow_{\text {raman level, and }}|g\rangle \rightarrow_{\text {ground level in Figure 1. Ion mass center }}$ is given with standard harmonic-oscillator of $H_{i o n}$ in $p_{x}=i \sqrt{\frac{1}{2} m v}\left(a^{+}-a\right)$ and $x_{i o n}=\sqrt{\frac{1}{2} m v}\left(a+a^{+}\right)$.

. Here, $a$ is annihilation operator and $a^{+}$creation operator of vibrational phonons. In Figure 1, laser frequencies are $\omega_{1}$ and $\omega_{2}$, and Rabi frequency is $\Omega$. Trapped ion-phonons total Hamiltonian is written 


$$
H=\left(\frac{\Omega}{2} e^{i \eta\left(a^{+}+a\right)}|e\rangle\left\langle g\left|+v a^{+} a-\delta\right| e\right\rangle\left\langle e\left|+\frac{\Omega}{2} e^{-i \eta\left(a+a^{+}\right)}\right| e\right\rangle\langle r|\right)+\text { ht.c. }
$$

here, LDP is $\eta=k / 2 m v, v$ is trap frequency of harmonic, and delta function is $\delta=v \eta^{2}$. We have taken the base vectors as follow:

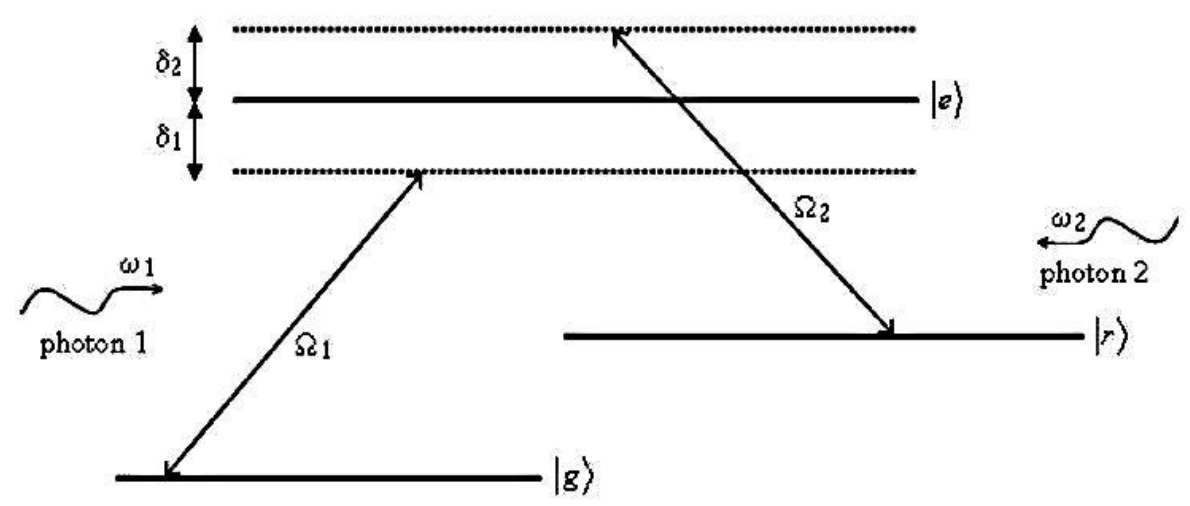

Figure 1. Three internal electronic levels of ion-phonons system. Time is given by dimensionless for the quantum system, time-independent parameters are allowed to be $\Omega=\Omega_{1}=\Omega_{2}, \omega=\omega_{1}=\omega_{2}$ and $\delta=\delta_{1}=\delta_{2}$.

$$
|e\rangle=\left(\begin{array}{l}
1 \\
0 \\
0
\end{array}\right),|r\rangle=\left(\begin{array}{l}
0 \\
1 \\
0
\end{array}\right),|g\rangle=\left(\begin{array}{l}
0 \\
0 \\
1
\end{array}\right)
$$

In this study, important transformed Hamiltonian is $\widetilde{H}=U^{+} H U$. Hamiltonian in Equation (4) is found after transmission action. $\Lambda$ model is given by a cascade $\Xi$ scheme in two phonons. Ion-two phonons system was covered by unitary transformation. Matrix of transformation, namely $U$ is performed [23],

$$
U=\frac{1}{2}\left(\begin{array}{ccc}
0 & \sqrt{2} & \sqrt{2} \\
-\sqrt{2} B[\eta] & B[\eta] & -B[\eta] \\
\sqrt{2} B[-\eta] & B[-\eta] & -B[-\eta]
\end{array}\right) .
$$

Here displacement operators of Glauber, $B(\eta)=e^{\left(i \eta\left(a+a^{+}\right)\right)}, B(-\eta)=e^{\left(-i \eta\left(a+a^{+}\right)\right)}$are achieved. $\widetilde{H}$ is performed $\widetilde{H}=\widetilde{H}_{0}+\widetilde{V}$, here

$$
\begin{gathered}
\widetilde{H}_{0}=v(|r\rangle\langle r|-| g\rangle\langle g|)+v \eta^{2}+v a^{+} a \\
\widetilde{V}=-i \frac{\sqrt{2} \delta \eta}{2}\left(a^{+}|e\rangle\left\langle r\left|-a^{+}\right| e\right\rangle\langle g|+\text { h.conjugate }\right)
\end{gathered}
$$

The LDR is performed between the values 0.006 and 0.08 of LDP. By using unitary transformation method [23], an initial state $|\psi(0)\rangle$ is written in following form 


$$
|\psi(t)\rangle=U_{0}^{+} U e^{-i t \widetilde{H}_{0}} K(t) U^{+}|\psi(0)\rangle,
$$

where $K(t)$ is typical vector for time-independent Hamiltonian; $e^{\left(-i t \widetilde{H}_{0}\right)}$ is the exponencial function, and $U_{0}=\exp (-i \omega t|e\rangle\langle e|)$ is the transformation matrix [23]. Trapped ion two phonon states system acts for $\Lambda$ scheme. The propagator is performed

$$
K(t)=\frac{1}{2}\left(\begin{array}{ccc}
\operatorname{Cos}(\Lambda t) & -\varepsilon S a^{+} & -\varepsilon S a \\
\varepsilon a S & 1+\varepsilon^{2} a G a^{+} & \varepsilon^{2} a G a \\
\varepsilon a^{+} S & \varepsilon^{2} a^{+} G a^{+} & 1+\varepsilon^{2} a^{+} G a
\end{array}\right),
$$

here $\varepsilon=v \eta / \sqrt{2}, \Lambda=\varepsilon \sqrt{2 a^{+} a+1}, \quad G=\frac{\operatorname{Cos}(\Lambda t)-1}{\Lambda^{2}}$ and $S=\frac{\operatorname{Sin}(\Lambda t)}{\Lambda}$. We take $v=10^{6} \mathrm{~Hz}$ and $\omega_{e g}=5 \times 10^{15} \mathrm{~Hz}$ for frequencies. In the system, we take $a=1$ and $b=0.005$. Normalization condition of ion is certainly $\left[\frac{1}{\sqrt{2}}\right]^{2}+\left[-\frac{1}{\sqrt{2}}\right]^{2}=1$, and normalization condition of two phonons is $\|a\|^{2}+\|b\|^{2}=|1|^{2}+|0.005|^{5} \cong 1$, approximately. So, the earliest of trapped ion-phonon states system is given as

$$
|\psi(0)\rangle=\frac{1}{\sqrt{2}}[|g\rangle-|r\rangle] \otimes(a|0\rangle+b|1\rangle),
$$

here, the phonon levels are $\langle 0|=(1,0)$, and $\langle 1|=(0,1)$. New equation for ion-two phonons is performed as

$$
|\psi(0)\rangle=\frac{1}{\sqrt{2}}[|g\rangle-|r\rangle] \otimes\left(\sum_{n=0}^{\infty} F_{n}(b)|n\rangle\right) .
$$

It is used by $\eta^{0}$ and $\eta^{1}$ are zero and first-order indication of LDP, for example. ion-two phonons system is evolved to an initial unentangled state,

$$
\left|\psi_{K}(t)\right\rangle=|\tilde{\psi}(0)\rangle=U^{+}|\psi(0)\rangle=\sum_{\sigma, m} N_{\sigma, m}(t)|\sigma, m\rangle .
$$

In Equation (11), ours system is produced in respect of $\sum_{\sigma, m} N_{\sigma, m}(t)|\sigma, m\rangle$. As a result of advanced mathematical transformations between Equation (9-13), the 12 probability amplitudes for the two quantum system are written 


$$
\begin{gathered}
N_{e 0}(t)=\left[\cos \left(\sqrt{\frac{1}{2}} t\right)+\frac{\eta i}{\sqrt{2}} \sin \left(\sqrt{\frac{1}{2}} t\right)\right] \exp [-t i / \eta] \\
N_{e 1}(t)=b \cos \left(\sqrt{\frac{3}{2}} t\right) \exp [-t i / \eta] \\
N_{e 2}(t)=-\frac{\eta i}{\sqrt{5}} \sin \left(\sqrt{\frac{5}{2}} t\right) \exp [-2 t i / \eta] \\
N_{r 0}(t)=\frac{b}{\sqrt{3}} \sin \left(\sqrt{\frac{3}{2}} t\right) \exp [-t i / \eta] \\
N_{r 1}(t)=\frac{\eta i}{\sqrt{2}}\left[\frac{3}{2}+\frac{2}{5} \cos \left(\sqrt{\frac{5}{2}} t\right)\right] \exp [-2 t i / \eta] \\
N_{g 3}(t)=-\frac{\sqrt{3}}{5} \eta i\left[1-\cos \left(\sqrt{\frac{5}{2}} t\right)\right] \exp [-2 t i / \eta] \\
N_{g 2}(t)=b \sqrt{\frac{2}{3}} \sin \left(\sqrt{\frac{3}{2}} t\right) \exp [-t i / \eta] \\
N_{g 1}(t)=\left[\sin \left(\sqrt{\frac{1}{2}} t\right)-\frac{\eta i}{\sqrt{2}} \cos \left(\sqrt{\frac{1}{2}} t\right)\right] \exp [-t i / \eta]
\end{gathered}
$$

and four amplitudes are zero: $N_{e 3}(t)=N_{r 2}(t)=N_{r 3}(t)=N_{g 0}(t)=0$. For Equations from (14) to (21), index $\sigma$ is positioned in the states of atomic $(g, r, e)$, index $m$ is positioned by vibrational numbers $(0,1,2,3)$. Vibrational phonon states are located by a Hilbert 4-space $\mathrm{H}_{p}$ and subsystem of trapped ionphonons is located in a Hilbert 3-space $\mathrm{H}_{i}$. Thus, two quantum system are in Hilbert $\mathrm{C}^{12}$-space. Here, $t$ is dimensionless and scaled with $v \eta_{\text {(harmonic trap frequency-LDP). What does }} v \eta_{\text {dimensionless }}$ mean? Accordingly in Figure 2, time 1 equals to 125 microsecond (ms). The mathematical calculation is as follows; for $v=1 \times 10^{6} \mathrm{Hertz}, \eta=0.08, v \eta=8 \times 10^{4}, \frac{1}{v \eta}=125 \times 10^{-6}=125 \mathrm{~ms}$. In the two quantum system, the final state vector is given by

$$
\left|\psi_{\text {final }}(t)\right\rangle=\sum_{m=0}^{3}\left(A_{m}(t)|e, m\rangle+B_{m}(t)|r, m\rangle+C_{m}(t)|g, m\rangle\right) .
$$


The coefficients $A_{m}(t) B_{m}(t)$ and $C_{m}(t)$ are shown by state vector amplitudes of $\Lambda$ and $\Xi$ models. These coefficients of the vector are

$$
\begin{gathered}
A_{m}(t)=\frac{1}{\sqrt{2}} e^{-i \omega t / v \eta}\left[N_{r m}(t)+N_{g m}(t)\right] \quad(m=0,1,2,3), \\
B_{0}(t)=-\frac{1}{\sqrt{2}} N_{e 0}(t)+\frac{1}{2} N_{r 0}(t)-\frac{i \eta}{2} N_{g 1}(t) \\
B_{1}(t)=-\frac{i \eta}{\sqrt{2}} N_{e 0}(t)-\frac{1}{2} N_{r 1}(t)+\frac{1}{2} N_{r 1}(t)-\frac{1}{2} N_{g 1}(t) \\
B_{2}(t)=-\frac{1}{\sqrt{2}} N_{e 2}(t)-\frac{i \eta}{\sqrt{2}} N_{g 1}(t)-\frac{1}{2} N_{g 2}(t) \\
C_{0}(t)=\frac{1}{\sqrt{2}} N_{e 0}(t)+\frac{1}{2} N_{r 0}(t)-\frac{i \eta}{2} N_{g 1}(t) \\
B_{3}(t)=-\frac{1}{2} N_{g 3}(t) \\
C_{1}(t)=-\frac{i \eta}{\sqrt{2}} N_{e 0}(t)+\frac{1}{2} N_{r 1}(t)+\frac{1}{2} N_{r 1}(t)-\frac{1}{2} N_{g 1}(t) \\
C_{2}(t)=\frac{1}{\sqrt{2}} N_{e 2}(t)+\frac{i \eta}{\sqrt{2}} N_{g 1}(t)-\frac{1}{2} N_{g 2}(t) \\
C_{g 3}(t)
\end{gathered}
$$

here $\omega_{e g}$ is frequency e-g levels, $\omega=\omega_{e g}-\eta^{2} v, i$ is complex number, and i is ion index. We plotted $N$ of two quantum system as $l \otimes l^{\prime}\left(l \leq l^{\prime}\right)$ in Figures 2, 3, 4 and Table 1. We found that final state vector $\left|\psi_{\text {final }}(t)\right\rangle$ is superposition of twelve function in Equations (23-31).

Hilbert spaces are $l=4$ for two-phonons, $l^{\prime}=3$ for ion. It is used a simplified density matrix $\rho_{\text {ion }}=\operatorname{Tr}_{\text {phonon }}\left(\rho_{\text {ion-p }}\right)$ by Equation (32). Fully density matrix $\rho_{\text {ion-p }}$ is performed with $12 \times 12$ matrix with respect to the bases $|i, p\rangle$. With tracing, $3 \times 3$-simplified density matrix, $\rho_{\text {ion }}$ is performed 


$$
\rho_{\text {ion }}=\operatorname{Tr}_{\text {phonon }}\left(\rho_{\text {ion-phonon }}\right)=\left(\begin{array}{lll}
\operatorname{Tr}|e\rangle\langle e| & \operatorname{Tr}|e\rangle\langle r| & \operatorname{Tr}|e\rangle\langle g| \\
\operatorname{Tr}|r\rangle\langle e| & \operatorname{Tr}|r\rangle\langle r| & \operatorname{Tr}|r\rangle\langle g| \\
\operatorname{Tr}|g\rangle\langle e| & \operatorname{Tr}|g\rangle\langle r| & \operatorname{Tr}|g\rangle\langle g|
\end{array}\right)
$$

where diagonal terms, $|e\rangle\langle e|| r\rangle,\langle r|$ and $|g\rangle\langle g|$ are a $4 \times 4$-matrix. For help to Equation (32), fully density matrix of two quantum system is written as:

$$
\rho_{\text {ion-phonon }}=(|Z\rangle\langle Z|)
$$

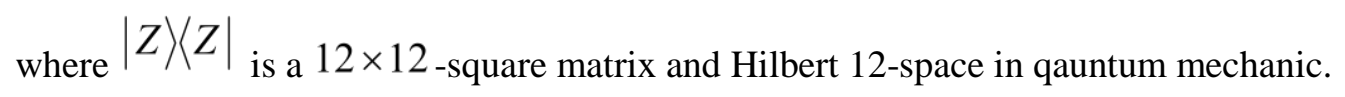

\section{THE MEASURE OF NEGATIVITY AND DISCUSSION}

The initial state in second section derive in Hilbert 12-space $H=H_{i} \otimes H_{p}$. In state vector $|\psi(t)\rangle$, fully density matrix of system is given by $\rho_{\text {ion-phonon }}=|\psi(t)\rangle\langle\psi(t)|=| Z\rangle\langle Z|$ in Equation(33). Negativity is first reported in literature as a quantum entanglement measurement in [20].

In this part, we examine if the state is entangled how much quantum entanglement it involves. It is analyzed quantum correlations with negativity [25]. The quantum state $\psi$ of a system such as $\mathrm{X}$ and $\mathrm{Y}$, with dimensions $k$ and $k^{\prime}$, can be given

$$
|\psi\rangle=\sum_{j} \sqrt{\mu_{j}}\left|x_{j}\right\rangle\left|y_{j}\right\rangle
$$

where $\sqrt{\mu_{j}},(j=1, \ldots, k)$ are Schmidt coefficients abbreviated as SCs, $x_{j}$ and ${ }^{y_{j}}$ are orthogonal basis in $H_{X}$ and $H_{Y}$ [25, 26]. We have given by Schmidt form for wave function. Therefore, three SCs are the three eigenvalues of the matrix in Equation (32), ${ }^{\mu_{j}}$ [26]. Their time dependence is illustrated in Figure 2. Upper two curves are $\mu_{1}$ and $\mu_{2}$, while the lower curve, ${ }_{3}$ is the third SCs for $\eta=0.08$ $\eta=0.06$ and $\eta=0.006$. Negativity of any quantum system is written as [26]

$$
\begin{gathered}
N(|\psi\rangle)=\frac{2}{k-1}\left(\sum_{i<j} \sqrt{\mu_{i}} \sqrt{\mu_{j}}\right) \\
N(|\psi\rangle)=\frac{2}{3-1}\left(\sqrt{\mu_{1}} \sqrt{\mu_{2}}+\sqrt{\mu_{1}} \sqrt{\mu_{3}}+\sqrt{\mu_{2}} \sqrt{\mu_{3}}\right)
\end{gathered}
$$


Dermez and Tunçer / Eskişehir Technical Univ. J. of Sci. and Tech. B - Theo.Sci. 8 (2)-2020

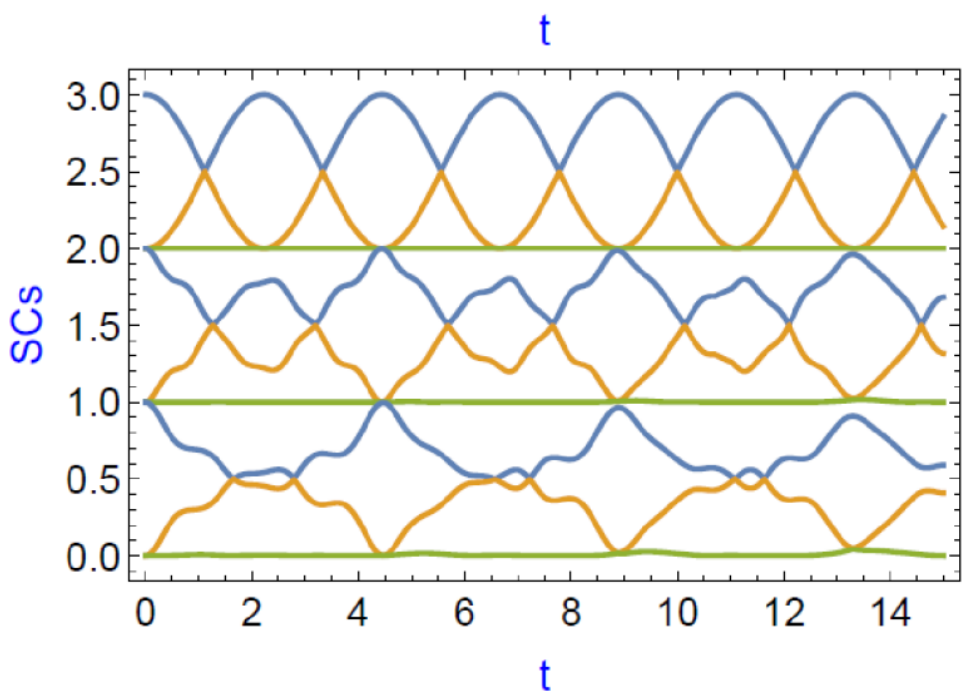

Figure 2. The time dependence of SCs, $\mu_{1}, \mu_{2}$ and $\mu_{3}$ for three LDP. Upper, middle and lower curves are for $\eta=0.08$ $\eta=0.06$ and $\eta=0.006$. The third SC, $\mu_{3}$ is gren and small. $\mathrm{t}$ is dimensionless scaled by $v \eta$. Earliest state of trapped ion and two phonons system is $\psi(0)=\frac{1}{\sqrt{2}}(|g\rangle-|r\rangle) \otimes(a|0\rangle-b|1\rangle)$ for $a=1, b=0.005$. These coupling parameters are written for $v=1_{\text {MHertz and }} \omega_{\text {eg }}=5 \times 10^{14} \mathrm{~Hz}$.
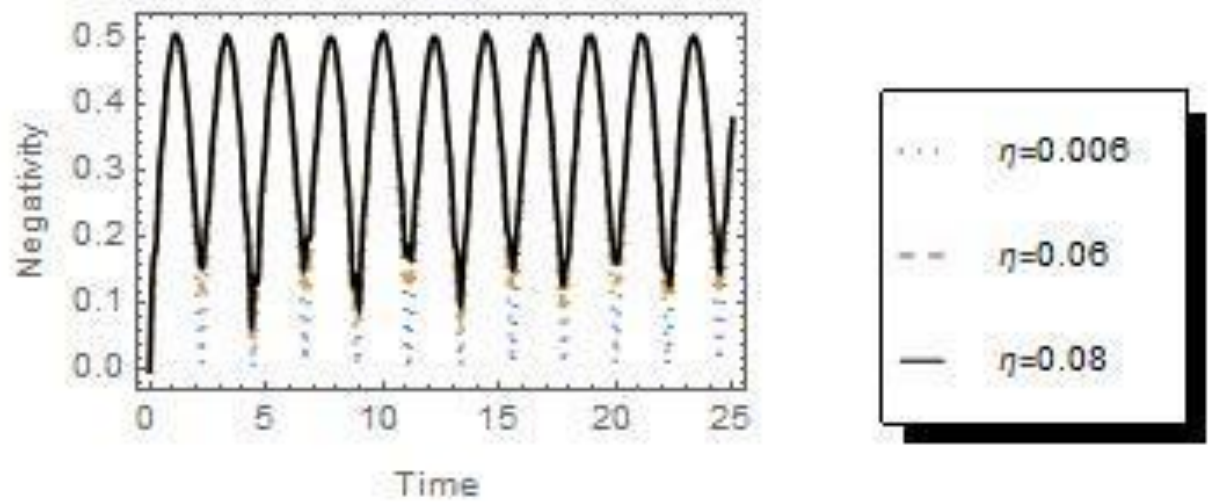

Figure 3. The time dependence of negativity, for three $\eta$. Three values are $\eta=0.08 \eta=0.06$ and $\eta=0.006$. Other assumptions parameters are the same as Figure 2 in the system.

Table 1. Six values of negativity within two ideal times, $t=4.0$ and $t=3.22$, with respect to Figure 3 .

\begin{tabular}{|c|c|c|c|}
\hline & $\begin{array}{c}\text { Fig } 3, \mathrm{a}=1, \mathrm{~b}=0.005, \\
\eta=0.006, v=1.0 \mathrm{MHz}\end{array}$ & $\begin{array}{c}\text { Fig } 3, a=1, b=0.005, \\
\eta=0.06, v=1.0 \mathrm{MHz}\end{array}$ & $\begin{array}{c}\text { Fig 3,a=1, b=0.005, } \\
\eta=0.08, v=1.0 \mathrm{MHz}\end{array}$ \\
\hline Negativity, $\mathrm{t}=4.0$ & 0.293 & 0.302 & 0.302 \\
\hline Negativity, $\mathrm{t}=3.22$ & 0.493 & 0.495 & 0.496 \\
\hline
\end{tabular}


This leads to higher dimensional entanglement with $\eta$. In Figure 3, time evolution of $N$ is illustrated by $\eta=0.006, \eta=0.06$ and $\eta=0.08$. We have obtained high amount of entanglement for three values of LDP.

Summerized, we obtained entanglement via negativity in the LDR discretely from other papers [16, 19]. The values of negativity in two ideal times are shown with Table 1. In Figures 2-3 and 4, a maximum value of negativity is reported $N=0.496$ for $\eta=0.08$ in Table 1 . The three values of $\eta$ are determined and taken into account throughout this study. In literature, we did not see that it has been worked with the value 0.08. We explain quantum dynamics of $N$ according to time in Figure 3 . The results of our former studies $[16,27,28]$ are in similar in Figures 3-4. N, C and E, which are the other advanced measurements defining entanglement motion, have been worked out in literature $[7,11,18,28,29,30]$.

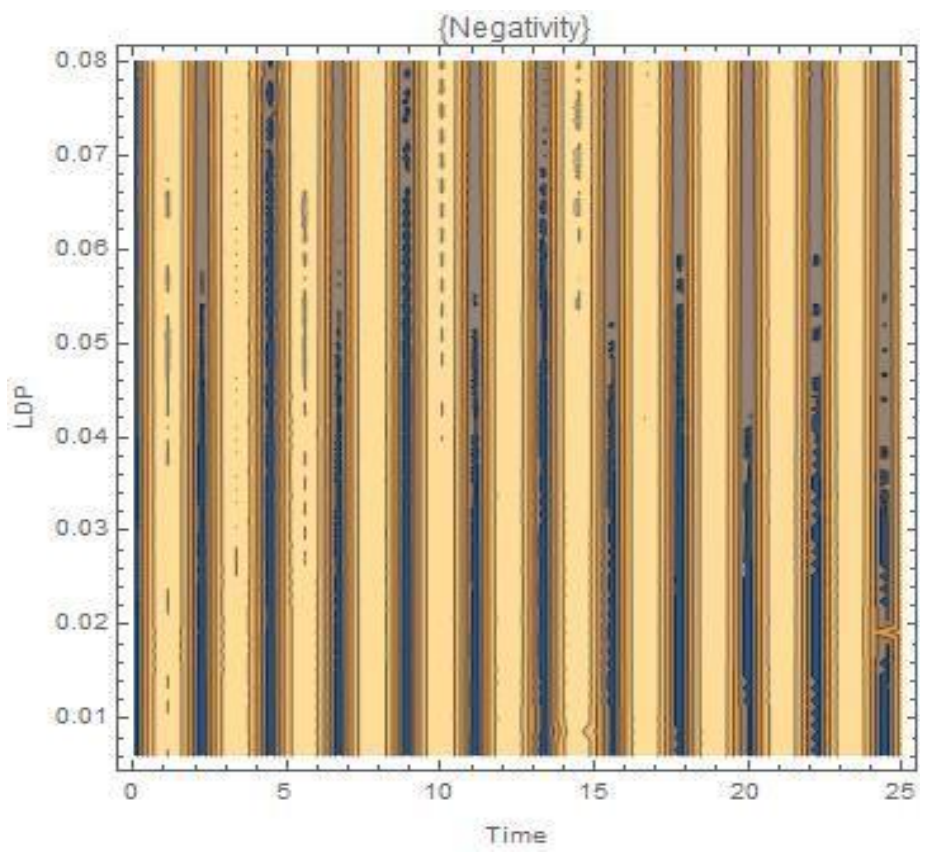

Figure 4. Contour plot of negativity as the time change of LDP (to $\eta=0.08$ from $\eta=0.006$ ). Color scale from black to orange equals to $0-1$ range. Other assumptions parameters are the same as Figure 2 in the system.

We show the quantum correlations with negativity for coupling parameters. We found seperate dynamic features in $N$ in reaction to increasing $\eta$. In Figure 3, $N$ oscillates between the values of minimum $N=0$ and highest rate $N=0.496$ at $\mathrm{t}=3.22$ for $\eta=0.08$. The variations between the maximum and the minimum values of negativity are regular with time. Time is maximally entangled state at optimum time point in Table 1. The presence of long lived entanglement in trapped ion and phonons system have been recognized by Figure 4 . We explore with $N$ that measurement degrees have a flash crop entangled state up in parallel to raising $\eta$ and this is in comparison to the previous observations [6, 27, 28, 29, 30,31]. 


\section{CONCLUSIONS}

We reported quantum entanglement of two quantum system in the Hilbert 12-space. We investigate the negativity through the definition of variance LDR. These plots are obtained by negativity with quantum corrections. Entanglement is compared and is analyzed by an quantum measure which is $N$. Quantum correlations and interactions between ion and two phonons is investigated. Because, the discussion on physical properties of trapped ion-two phonos interaction is an important subject for quantum information.

Concluding remarks are; (1) in our system, quantum entanglement is shown to have the capacity and degree of negativity is $N=0.501$; (2) $N$ bases on three different LDPs; (3) This extracts that such entanglement is connected with $\eta$. We achieved long-lived entanglement in LDR. Maximally entangled states as presented by means of ion-two phonons system can be important for researchers with trapped ions. Extending the life time can be succeed by using Rabi frequences and $\eta$. This study and similar studies based on quantum measurement will lead to a better understanding of quantum theory and entanglement.

\section{ACKNOWLEDGMENTS}

This work is supported by University of Afyon Kocatepe 18-Kariyer.64 project.

\section{REFERENCES}

[1] Heisenberg W. The Physical Principles of the Quantum Theory. Dover Publications, New York, NY, USA, 1930.

[2] Einstein A, Podolsky B, Rosen N. Can quantum-mechanical description of physical reality be considered complete? Phys. Rev. 1935; 47: 777-780.

[3] Bohr N. Can quantum-mechanical description of physical reality be considered complete? Phys. Rev. 1935; 48: 696-702.

[4] Schördinger E. Die gegenwrtige situation in der quantenmechanik. Naturwissenschaften. 1935; 23: 807-812.

[5] Hitoshi I. Quantum measurement cannot be a local physical process, journal of quantum information science. 2019;9:171-178.

[6] Landsman KA, Wu Y, Leung PH, Zhu D, Linke NM, Brown KR, Duan LM, Monroe C. Twoqubit entangling gates within arbitrarily long chains of trapped ions. Phys. Rev. A. 2019; 100: 02:2332.

[7] Muhammed A. Qualitative aspects of the entanglement in the three-level model with photonic crystals. App. Phys. B. 2005; 81; 193-203.

[8] Wong-Campos JD, Moses SA, Johnson KG, Monroe C. Demonstration of two-atom entanglement with ultrafast optical pulses. Phys. Rev. Lett. 2017; 119: 23: 0501. 
Dermez and Tunçer / Eskişehir Technical Univ. J. of Sci. and Tech. B - Theo.Sci. 8 (2)-2020

[9] Wootters WK. Entanglement of formation of an arbitrary state of two qubits. Phys. Rev Lett. $1998 ; 80 ; 2245-2248$.

[10] Guha MM. Quantum information processing using the exchange interaction. Journal of Quantum Information Science. 2018; 8; 139-160.

[11] Dermez R, Deveci B, Güney DO. Quantum dynamics of a three-level trapped ion under a timedependent interaction with laser beams. Eur. Phys. J. D. 2013; 67: 120.

[12] James DFV. Quantum computation and quantum information theory. Appl. Phys. B. 1998; 66: 181-190.

[13] Monreo C, Meekhof DM, King BE, Wineland DJ. A Schrödinger cat superposition state of an atom science. 1996; 272: 5265: 1131-1136.

[14] Dermez R, Özen S. Higher dimensional entangled qudits in a trapped three-level ion. Eur. Phys. J. D. 2010;57: 431-437.

[15] Yu GY, Du S, Li X, Wu S. Entangled bases with fixed Schmidt number. Journal of Physics A: Mathematical and Theoretical. 2015; 48: 24: 245301.

[16] Dermez R, Müstecaplıoğlu ÖE. Long-lived entangled qudits in a trapped three-level ion beyond the Lamb-Dicke limit. Phys. Scr. 2009; 71: 015304.

[17] Nieuwenburg E, Zilberberg O. Entanglement spectrum of mixed states. Phy. Rev. A. 2018; 98 : 012327.

[18] Neumann J. Mathematical Foundations of Quantum Mechanics. Princeton Uni. Press, NJ, USA: 1995.

[19] Dermez R. Concurrence and negativity as a family of two measures elaborated for pure qudit states. Journal of Russian Laser Research. 2017; 38: 408-415.

[20] Zheng SB, Kuang LM, Gao KL. Two-mode squeezed states and their superposition in the motion of two trapped ions. Phys. Lett. A. 2002; 300, 427-431.

[21] Zheng SB. Preparation of motional macroscopic quantum-interference states of a trapped ion. Phys. Rev. A. 1998; 58: 761.

[22] Zheng SB. Control of motional states of a trapped ion. Phys. Lett. A 1998; 248, 25-28.

[23] Müstecaplıoğlu ÖE. Motional macroscopic quantum superposition states of a trapped three-level ion. Phys. Rev. A. 2003; 68: 023811.

[24] Sakurai JJ. Modern Quantum Mechanics. Addison-Wesley Publishing Company, USA: 1994.

[25] Vidal G, Werner RF. Computable measure of entanglement. Phys. Rev. A. 2003; 65: 032314.

[26] Lee S, Chi DP, Oh SD, Kim J. Convex-roof extended negativity as an entanglement measure for bipartite quantum systems. Phys. Rev. A. 2003; 68: 062304. 
Dermez and Tunçer / Eskişehir Technical Univ. J. of Sci. and Tech. B - Theo.Sci. 8 (2)-2020

[27] Dermez R. Generalized concurrence and negativity in time-dependent C3 $\otimes C 5=\mathrm{C} 15$ dimensional ionic-phononic systems. Journal of Russian Laser Research. 2016; 37: 572-580.

[28] Dermez R. Comparing concurrence and negativity in time-dependent ionic-phononic system with fifteen dimensional density matrix. IOP Publishing Journal of Physics: Conference Series 766, 2016: 012012.

[29] Anvar SJ, Ramzan M, Khan MK. Dynamics of entanglement and quantum Fisher information for N-level atomic system under intrinsic decoherence. Quantum Information Process. 2017; 16:142.

[30] Liao QH, Wu JF, Wang P. Properties of entanglement between the two trapped ions. Indian J. Phys. 2017; 91: 12: 1615-1624.

[31] Dermez R., Analyzing of Quantum Entanglement with Concurrence in the Deep Lamb-Dicke Regime, Adiyaman University Journal of Science, 2020; 10(1), 339-352. 